\title{
Eine Praxis für Unsichtbare
}

\section{Erhard Taverna}

Die Schätzungen gehen auseinander, sie reichen von 100000 bis zu 200000 Menschen, die mitten unter uns leben, obwohl sie offiziell gar nicht vorhanden sind. Administrativ gibt es sie nicht, denn sie haben keine gültige Aufenthalts- und Arbeitsbewilligung. Man findet sie, in der Mehrzahl weiblich, von ausserhalb Europas, als Putzfrauen, Altenbetreuerinnen und Babysitter, auf Baustellen und in der Landwirtschaft, im Gastund im Sexgewerbe. Sie leben im Dauerstress, haben Angst vor Ausweisung, werden vielfach ausgebeutet und sind vom Gesundheitswesen ausgeschlossen. Die «Médecins Sans Frontières», bekannt für ihre Einsätze in Konflikt- und Krisenregionen, starteten ihr erstes Projekt einer Schattenmedizin für «Sans-Papiers» in Freiburg und eröffneten 2006 die medizinische Beratungs- und Behandlungsstelle «Meditrina» in Zürich (www.msf.ch/meditrina).

David Winizki, geboren 1948, gehört zu den Pionieren eines Netzwerkes von rund 25 Allgemeinmedizinern und 15 Spezialisten, die mit Zahnärzten, Apothekern, Labors und weiteren nichtärztlichen Berufen den Gesundheitstreffpunkt in der Anwandstrasse 7 unterstützen. David Winizki durchlief nach dem Staatsexamen in

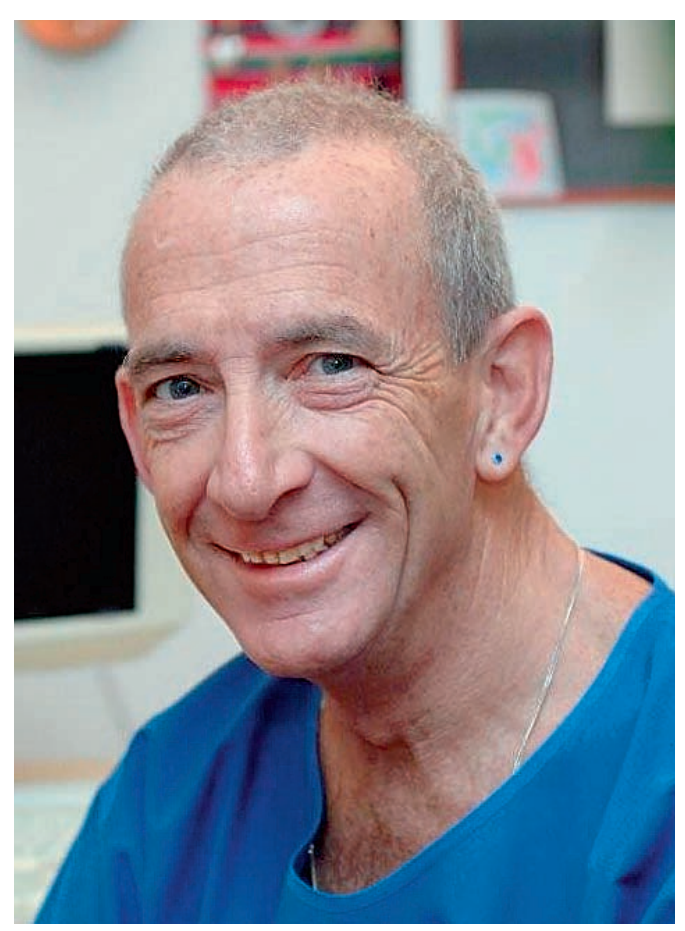

David Winizki, Arzt der medizinischen Beratungsund Behandlungsstelle «Meditrina» in Zürich.
Zürich seine Weiterbildung zum Facharzt für Allgemeinmedizin und eröffnete 1989 seine Praxis, die er im Tiefenbrunnen zusammen mit einem Kollegen führt.

Durch den Eingang im Erdgeschoss einer ehemaligen Schreinerei komme ich direkt ins Wartezimmer, wo drei wartende Frauen miteinander spanisch sprechen. Südamerikanerinnen machen mehr als die Hälfte der Patientinnen aus. Drei Viertel von ihnen sind nie irgendwo registriert gewesen, sie arbeiten im Untergrund für durchschnittlich 1500 Franken im Monat. Rund ein Viertel war irgendwann einmal registriert und ist danach untergetaucht, sei es weil ein Asylgesuch abgelehnt oder wegen fehlender Papiere Nichteintreten beschlossen wurde oder weil eine Heirat vor fünf Jahren durch Scheidung oder Tod des Partners endete. Da sie häufig keinen Job finden, sind sie am schlechtesten dran. Die häufigsten Probleme sind psychosomatischer Art, Schmerzen der Bewegungsorgane, Depressionen, gynäkologische Leiden und unerwünschte Schwangerschaften.

Für viele Frauen bleibt die Heirat mit einem Einheimischen die einzige Chance, um dem Schattendasein zu entrinnen, transkulturelle Beziehungen sind aber krisenanfällig. Eine Investition von 20000 bis 30000 Franken, was sich meistens nur Prostituierte leisten können, ist riskant. Als Opfer von Zuhältern und erpresserischen Männern riskieren sie das Scheitern einer Ehe, die als Papierheirat verboten ist und erst nach fünf Jahren zur eheunabhängigen Daueraufenthaltsbewilligung führt. Gewalt, Vergewaltigungen und Lohnbetrügereien gehören dabei zum täglichen Risiko. Auch Papierlose haben Anspruch auf eine Krankenkasse und Prämienverbilligung, doch selbst in Genf, wo der Kanton niederschwellige Angebote finanziert, lassen sich höchstens 5-10\% der Untergetauchten, aus Angst vor polizeilichen Überprüfungen, versichern. Eine anonymisierte Versicherungskarte gibt es nicht, dafür eine Grundversorgung ohne Angabe der Wohnadresse, selbst bei stationären Aufnahmen im Triemlispital, das zudem nur den Spitalanteil, ohne Kantonsbeitrag, in Rechnung stellt.

Die Schattenmedizin existiert mit stiller Duldung und Förderung des kantonalen Gesundheitsdepartementes oder der Stadt, die im «Ambulatorium Kanonengasse» eine Anlaufstelle für 
Obdachlose und Illegale finanziert. Auch die Krankenkassen zeigen sich einsichtig und sind bereit, Kranke aufzunehmen, die erst mit Behandlungsbeginn gemeldet werden. Auch was politisch und behördlich nicht existiert, folgt hier einer eigenen Logik. Mit der Ratifizierung des UNO-Paktes für soziale und kulturelle Menschenrechte hat die Schweiz den Anspruch auf Gesundheit und Schulung der Kinder anerkannt. Nichtstaatliche Organisationen, Hilfswerke, Kirchen und Private widmen sich den real existierenden Problemen von Menschen, die ohne diese Hilfe die übrige Bevölkerung sozial und medizinisch gefährden würden und zudem oft unentbehrliche Arbeiten verrichten. Seit den 90er Jahren hätten die Armutsprobleme deutlich zugenommen, sagt der Praktiker. Selbst Schweizer und Schweizerinnen, die ihre Kassenprämien oder hohen Franchisen nicht zahlen könnten, benützten zunehmend das Netzwerk von Meditrina.

Dass dieser Pakt der Vernunft überhaupt zustande kam, ist Pionieren wie David Winizki zu verdanken. Auch Idealisten müssen leben. Die meisten Patienten sind krankenversichert, die übrigen zahlen bar, 50 Franken für eine Konsultation, Praxislabor und alle nötigen Unter- suchungen, wie zum Beispiel Ultraschall, inbegriffen. Zahlreiche kleine Geschenke auf dem Fenstersims zeugen von dankbaren Patientinnen, die hier einen kompetenten, mehrsprachigen und zuverlässigen Arzt gefunden haben. Oft kommen Angehörige mit, und wenn sie zufrieden sind, bleiben sie der Praxis über weite Distanzen und viele Jahre treu. Aber auch aus dem Quartier kommen Patienten, die einen guten Hausarzt zu schätzen wissen.

David Winizki war aktiv an der «DrolegInitiative» beteiligt und gehört zu den Begründern der «Sans-Papiers-Anlaufstelle Zürich» SPAZ (www.s-paz.ch), die von Gewerkschaften und MigrantInnenorganisationen aufgebaut wurde. Sie bietet allen Illegalen in der Region Zürich an zwei Wochentagen im Volkshaus Informationen und Beratung sowie Kontakte zu Juristen, Medizinern und anderen Stellen.

Der Rundgang zeigt einfache, aber zweckmässig eingerichtete Praxisräume. Auffallend sind die vielen Stadtkarten und Quartierpläne an den Wänden. David Winizki sammelt seit Jahren diese Dokumente, doch nicht zur eigenen Orientierung, denn dafür folgt er seinen persönlichen Koordinaten einer aktiven Politik und engagierten Menschlichkeit. 